\title{
Fitoestrógenos dietarios y sus potenciales beneficios en la salud del adulto humano
}

\author{
Argelia Garrido Ga, María Pía de la Maza C, \\ Luis Valladares $B^{\mathbf{b}}$. \\ Potential health benefits of \\ phytoestrogens
}

Human diet contains a series of bioactive vegetal compounds that can improve human health. Among these, there has been a special interest for phytoestrogens. This article reviews the evidence about the potential benefits of phytoestrogens for human health. Forty eight manuscripts were selected for their study design and relevance to human health. The cell growth inhibitory effects of phytoestrogens and their implication in breast cancer are reviewed. Also the effects of these compounds on serum lipid levels and the effectiveness of a phytoestrogen derivate, ipriflavone, on the prevention of osteoporosis are analyzed. Although these compounds have a great potential for improving health, there is still not enough evidence to recommend the routine use of phytoestrogens (Rev Méd Chile 2003; 131: 1321-28). (Key Words: Breast neoplasms; Isoflavones; Lipids and antilipemic agents; Phytoestrogen)

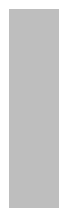

Recibido el 24 de abril, 2003. Aceptado en versión corregida el 8 de septiembre, 2003. Financiamiento: FONDECYT \# 1000946.

Laboratorio de Hormonas-Receptores y Laboratorio de Lípidos-Antioxidantes. Instituto de Nutrición y Tecnología de los Alimentos, Universidad de Chile.

aMagíster en Nutrición Humana

${ }^{\mathrm{b}}$ Doctor en Bioquímica

$E^{n}$ la actualidad la expectativa de vida del ser humano es de 75 a 78 años y puede incrementar a 85 años en las próximas dos décadas; sin embargo, no es claro si pueda ser satisfactorio vivir estos años adicionales. Numerosos estudios indican que el incremento en años de vida no siempre está asociado a una mejor calidad de la misma, dados los cambios físicos, mentales y sociales que se desencadenan con la edad. Muchas de las enfermedades propias de las socieda-

Correspondencia a: Dr. Luis Valladares. Macul 5540. Santiago de Chile. Fax: 2214030.

E mail: ivallada@uec.inta.uchile.cl des occidentales son dependientes de las hormonas y los estudios epidemiológicos han llevado a establecer una estrecha asociación entre la incidencia de enfermedades tales como cáncer de próstata, de mama, de colon, enfermedades cardiovasculares (ECV), con la carencia de cierto tipo de vegetales en la dieta ${ }^{1-3}$. Es extremadamente difícil identificar los componentes de la dieta útiles para prevenir el riesgo de las enfermedades mencionadas anteriormente. Los estudios de intervención de la dieta, que generalmente son de corto tiempo, no necesariamente pueden reflejar las respuestas observables en estudios de largo plazo, sin embargo, las investigaciones en nutri- 
ción han podido establecer los beneficios que para la salud puede generar una intervención dietaria $^{4}$. Se ha podido establecer que la dieta humana contiene, además de los macro y micro nutrientes, una serie de compuestos no nutrientes bioactivos, de origen vegetal, que pueden ser importantes para la salud, conocidos como fitoquímicos. Entre éstos, los fitoestrógenos constituyen un grupo de compuestos no esteroidales, que pueden comportarse como agonistas o antagonistas de los estrógenos. Los principales fitoestrógenos con importancia en nutrición y que pueden tener relevancia sobre la salud humana son las isoflavonas, los lignanos y los cumestanos, siendo especialmente activas las primeras ${ }^{5}$.

Las isoflavonas se encuentran en una variedad de plantas, incluyendo frutas y vegetales, aunque es el poroto de soya el que presenta las más altas concentraciones (Tabla 1), en las formas de glicósidos, genistina, daidzina y glicitina de sus correspondientes formas agliconas, genisteína, daidzeína y gliciteína (Figura 1) ${ }^{6}$. El contenido de isoflavonas en el poroto de soya sin procesamiento es de aproximadamente $1 \mathrm{mg} / \mathrm{g}$ con un rango de $0,4 \mathrm{a}$ $2,4 \mathrm{mg} / \mathrm{g}^{7}$. Las isoflavonas glicosiladas ingeridas son hidrolizadas a las formas agliconas genisteína, daidzeína y gliciteína por bacterias en el intestino delgado, donde la daidzeína puede ser metabolizada, también por bacterias, a otras dos formas de isoflavonas: equol (que presenta actividad estrogénica) y O-desmetilangolensina (sin actividad estrogénica). Las agliconas son absorbidas por la mucosa intestinal y transportadas por la vena porta al hígado. Aquí, por acción de las enzimas glucoroniltransferasas y sulfotransferasas forman conjugados de glucoronidos y sulfatos, siendo la glucoronidación la principal vía de biotransformación de las isoflavonas ${ }^{8}$. Estas etapas de biotransformación son de importancia en la acción de las

\section{Tabla 1. C ontenido de isoflavonas en algunos alimentos}

\begin{tabular}{|lc|}
\hline Alimento & $\begin{array}{c}\text { Isoflavonas } \\
\text { totales } \\
\text { (mg/100g) }\end{array}$ \\
\hline Poroto de soya & 153 \\
Harina de soya & $148-178$ \\
Leche de soya (polvo) & 194 \\
Salsa de soya & $0,1-1,6$ \\
Tofu & 28 \\
Miso & 42,5 \\
Té verde (Japón) & 0,05 \\
Porotos (diferentes variedades) & $0,21-0,74$ \\
\hline
\end{tabular}

*Base de datos de USDA-Iowa State University

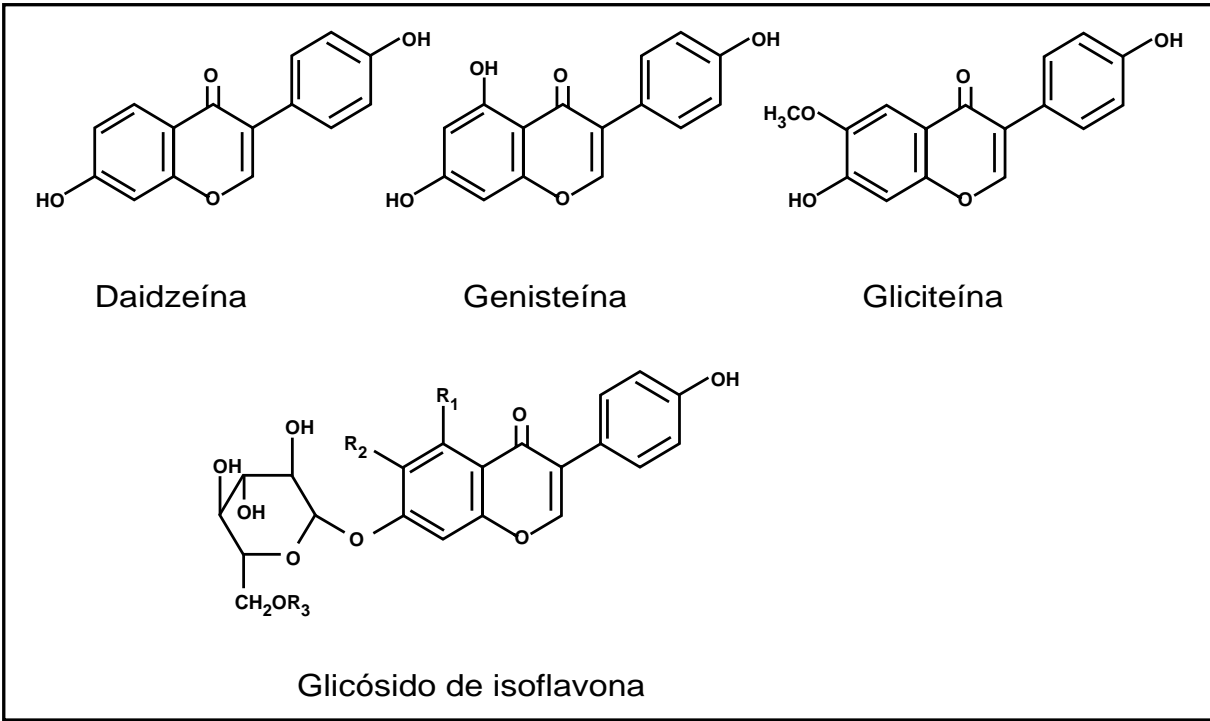

FIguRA 1. Estructura de las isoflavonas de la soya y del glicósido de isoflavona. $R_{1}, R_{2}$ y $R_{3}$ corresponden a $H$, excepto en genistina $R_{1}=O H$ y en glicitina $R_{2}=O C H 3$. 
isoflavonas, como también la metabolización de daidzeína a equol ${ }^{9}$. Varios estudios, incluyendo los de nuestro laboratorio, han mostrado que variaciones individuales podrían ser importantes para determinar la biodisponibilidad y la ruta de metabolización de las isoflavonas ${ }^{10,11}$. Dada la dificultad para medir las concentraciones plasmáticas de las isoflavonas, la biodisponibilidad de estos compuestos sigue siendo uno de los temas menos comprendidos y de mayor controversia en la interpretación de los resultados. Los niveles de isoflavonas en el suero son dependientes del consumo de alimentos ricos en soya o de la administración de isoflavonas como suplemento. El nivel plasmático de isoflavonas en poblaciones asiáticas es alrededor de 0,5 $\mu \mathrm{M}$, en cambio en Chile la concentración de isoflavonas es de aproximadamente $0,02 \mu \mathrm{M}^{10,13}$. En un estudio farmacocinético con ingesta de agliconas purificadas, la mayor concentración plasmática se alcanza a las 5 a $6 \mathrm{~h}$ y la eliminación desde el plasma tiene una vida media de 6 a $8 \mathrm{~h}^{14}$. La biodisponibilidad de las isoflavonas es mayor cuando se ingieren como ß-glicósidos que en forma de agliconas ${ }^{14,15}$. Los estudios de farmacocinética y biodisponibilidad muestran que se obtienen niveles plasmáticos más altos con una ingesta repetida durante el día, como también el exceso de ingesta de isoflavonas conduce a una disminución de la absorción. Las isoflavonas circulan en el plasma principalmente en la forma conjugada, mayoritariamente unidas a ácido glucorónico y menos de 3\% circula en la forma libre. Los estudios sobre los efectos biológicos de las isoflavonas se han dirigido casi exclusivamente a las formas no glicosiladas: genisteína, daidzeína y equol; sin embargo, el trabajo de Zhang ${ }^{16}$ plantea que las formas conjugadas de genisteína y daidzeína podrían tener relevancia en nutrición y un impacto en patologías dependientes de hormonas. Poca atención se ha dado en la literatura a los conjugados sulfato de las isoflavonas, aunque hay datos que muestran su posible actividad biológica. Por ejemplo, el sulfato de daidzeína inhibe competitivamente a las sulfatasas de esteroles en microsomas de hamster. Así como los sulfatos de esteroides son hidrolizados por las sulfatasas, también los derivados de isoflavona podrían originar agliconas activas en algunos tejidos.

Desde una perspectiva biológico-funcional, la estructura difenólica de la genisteína (4',5',7-trihi- droxi-isoflavona) y de la daidzeína (4',7'-dihidroxiisoflavona) es importante, ya que la presencia de los grupos hidroxilos en sus moléculas, como también de los anillos aromáticos, les permite interactuar con el receptor para estradiol, manifestando respuestas como agonistas o antagonistas estrogénicos ${ }^{17}$. La acción de las isoflavonas a nivel celular y molecular puede modificarse por factores como la concentración del ligando, los niveles endógenos de estrógenos u otros esteroides, la presencia de reguladores transcripcionales y por el tipo de receptores intracelulares de estradiol [R $\alpha$ y Rß]. La distribución selectiva anatómica del receptor $\mathrm{R} \Omega$ en tejidos como hueso, cerebro, endotelio vascular y plaquetas proporciona un paradigma para comprender la respuesta de estos compuestos con actividad estrogénica ${ }^{18}$. La genisteína y la daidzeína son agonistas estrogénicos débiles con respecto al $\mathrm{R} \alpha$, con una potencia relativa referente a 17ß-estradiol de $8 \%$ y $3 \%$, respectivamente; en cambio, con respecto al $\mathrm{R} ß$ la genisteína tiene una potencia relativa de $36 \%{ }^{19}$. Dada la afinidad de las isoflavonas por los $R \alpha$ y $\mathrm{R} ß$, la participación de estos compuestos en procesos fisiológicos podría considerarse muy parcial; sin embargo, sus acciones pueden alcanzar relevancia, considerando que las concentraciones plasmáticas pueden ser del orden de 10.000 a 20.000 veces la concentración del estradiol circulante. Uno de los aspectos más importantes de la farmacología molecular ha sido el desarrollo de los moduladores selectivos de los receptores de estrógenos (SERMs): tamoxifeno y raloxifeno. Estos pueden actuar como antagonistas del estradiol en la mama y el endometrio y como agonistas del estradiol en el cerebro, endotelio vascular y hueso ${ }^{20}$. Los estudios moleculares de cristalografía con el receptor $R ß$, demuestran que la posición espacial que adopta el complejo genisteína- Rßreguladores transcripcionales, es similar al que adopta el $\mathrm{R} ß$ al interactuar con el raloxifeno ${ }^{21}$. De esta manera, las isoflavonas más que estrógenos pueden ser consideradas como SERMs naturales ${ }^{22}$.

Además de la acción de las isoflavonas por interacción con los receptores del estradiol, estos compuestos pueden actuar fisiológicamente a través de: 1 . Inhibición de enzimas que participan en el metabolismo de los esteroides: estrógeno sintetasa (aromatasa), tirosina quinasa, $5 \alpha$ - reductasa, sulfatasa, sulfotransferasa, 17ß-hidroxi-esteroide de- 
hidrogenasa y la 3ß-hidroxi-esteroide dehidrogena$\mathrm{sa}^{23}$. 2. Estímulo de la síntesis de la globulina que une hormonas sexuales (SHBG) ${ }^{10,24}$. 3. Inhibición de la producción de radicales libres del oxígeno (propiedades antioxidantes) ${ }^{25}$.

La posibilidad de que las isoflavonas puedan ejercer varias de las acciones propuestas para los estrógenos, especialmente con relación a las enfermedades cardiovasculares y la osteoporosis, pero sin las desventajas frente al cáncer de mama y de endometrio, ha llevado a muchas mujeres a considerar el consumo de soya como una alternativa a la terapia de reemplazo hormonal.

\section{FITOESTRÓGENOS Y SUS IMPLICANCIAS CLINICAS}

Prevención de cáncer: Las isoflavonas tienen potencialidad anticancerígena. Los primeros estudios se centraron en la actividad estrogénica, particularmente en la capacidad para reducir el riesgo de cáncer de mama, posteriormente los estudios han revelado que sus efectos sobre la prevención del cáncer pudieran estar mediados por mecanismos no hormonales. Los mecanismos propuestos para estas acciones incluyen: 1. Inhibición de DNA topoisomerasas; 2 . Supresión de la angiogénesis; 3. Inhibición de la inducción a diferenciación en líneas celulares de cáncer; y 4. Inducción de la apoptosis $^{26}$. En animales de experimentación, un metaanálisis mostró que la administración de productos de soya produjo una disminución en la incidencia 0 la multiplicidad de tumores en modelos de cáncer de mama, próstata, hígado, esófago y pulmón ${ }^{27}$.

En muchos estudios, la atención se ha focalizado sobre la genisteína, sustancia que sería el principal anticancerígeno presente en la soya, por sus propiedades antioxidantes y como inhibidor de las proteínas tirosina-quinasas ${ }^{28}$. Sin embargo, son necesarias algunas precauciones en la interpretación de las evidencias actuales. Gran parte de los efectos inhibitorios sobre el desarrollo de tumores se ha obtenido con dosis muy altas de fitoestrógenos (10-50 $\mu \mathrm{M})$, no comparables con las que pueden obtenerse a través de la dieta. En este aspecto, en la actualidad no es posible establecer ni la dosis ni el tipo de fitoestrógenos que debería usarse en la supresión de tumores, como tampoco la frecuencia ni la duración de su uso, sus efectos potenciales reales y su toxicidad. Hasta este momento, al menos en nuestro conocimiento, no hay protocolos en humanos acerca del uso de soya o sus derivados en la prevención primaria 0 secundaria del cáncer. La incidencia del cáncer de mama varía en diferentes comunidades en el mundo y los estudios de migración de poblaciones sugieren que estas diferencias se explicarían mejor por factores ambientales que por condiciones genéticas. Es así como las mujeres asiáticas tienen incidencias del orden de un tercio a 50\% menores que en mujeres occidentales. Algunos estudios de población han sugerido que hay una asociación inversa entre el consumo de soya por las poblaciones orientales y el cáncer de mama. En el estudio Shanghai de cáncer de mama, que se realizó con mujeres residentes en esa ciudad, que fueron diagnosticadas en un período de dos años, se demostró una fuerte asociación inversa entre el consumo de alimentos de soya durante la adolescencia y el riesgo de cáncer de mama en la vida adulta ${ }^{29}$. Los estudios realizados en países de occidente también demuestran que la ingesta de soya protege del cáncer mamario, aunque la ingesta de soya en estas poblaciones es muy baja. En estos estudios la relevancia biológica de la correlación inversa entre ingesta de soya y riesgo de cáncer mamario puede tener un significado cuestionable, ya que podría también indicar que la mujer occidental típica que consume soya tiene además un estilo de vida que la protegenía contra el cáncer mamario ${ }^{30}$.

No es posible dar recomendaciones sobre el uso de fitoestrógenos en la prevención o tratamiento del cáncer. Sin embargo, el hecho de que la genisteína pueda actuar más como un SERMs que como un estrógeno, apoya el planteamiento de que los alimentos con soya o suplementos de isoflavonas pueden ser beneficiosos en la prevención y en el tratamiento del cáncer, como lo ha demostrado un estudio con raloxifeno ${ }^{31}$.

Osteoporosis. La terapia de reemplazo estrógenico es altamente efectiva en reducir la velocidad de pérdida de hueso como también la remodelación del mismo ${ }^{32}$. Los datos epidemiológicos sugieren que la menor osteoporosis observada en las mujeres asiáticas, cerca de un tercio de lo 
observado en la mujer occidental, sería consecuencia de un alto consumo de soya. En este aspecto, Kardinaal y $\mathrm{col}^{33}$ proponen que la velocidad de pérdida de hueso está inversamente asociada con la excreción urinaria de fitoestrógenos. Por otro lado, en modelos con animales se han demostrado los posibles efectos positivos de las isoflavonas sobre la pérdida de hueso ${ }^{34}$. Los estudios se han centrado principalmente en la ipriflavona, compuesto no hormonal producido sintéticamente a partir de la daidzeína ${ }^{35,36}$. La ipriflavona no actuaría directamente sobre los receptores de estrógenos y, en este aspecto, estrictamente hablando no sería un fitoestrógeno; sin embargo, aproximadamente $10 \%$ de la ipriflavona puede ser convertida a daidzeína. Por otro lado, hay algunas evidencias de que el tratamiento con ipriflavona puede también incrementar la densidad mineral ósea. En general, el uso de la ipriflavona parece seguro, sin embargo, en la mayor parte de los estudios se han analizado sólo hasta 2 años de tratamiento. Dado que la ipriflavona no presenta efectos estrogénicos, este compuesto puede representar menor riesgo para los cánceres dependientes de estrógenos, pero por otro lado sus efectos a nivel cardiovascular estarían disminuidos.

Enfermedades cardiovasculares: Las enfermedades cardiovasculares (ECV) son causa importante de muerte en los países occidentales y la dieta tiene un impacto sobre los factores de riesgo, como la hipercolesterolemia, hipertrigliceridemia, colesterol-LDL, colesterol-HDL, obesidad, diabetes. Además de los factores dietarios recomendados para disminuir los riesgos cardiovasculares, se propone que el consumo de soya podría otorgar beneficios adicionales. Se han realizado numerosos estudios de intervención dietaria, usando alimentos que contienen isoflavonas derivadas de soya, para observar la disminución del colesterol circulante y del riesgo de enfermedades cardiovasculares. En 1999 la agencia estatal para la Administración de Alimentos y Drogas (FDA) de los Estados Unidos permitió el uso de un "health claim" para la proteína de soya, asociando el consumo de esta proteína y una dieta baja en grasas saturadas, con la disminución del riesgo de enfermedades cardiovasculares. Esta medida se basó en los estudios incluidos en el metaanálisis de 38 estudios clínicos controlados en que se usó proteína de soya ${ }^{37}$. En este metaanálisis se concluyó que la sustitución de proteína animal por proteína de soya baja significativamente el colesterol total, el colesterol-LDL y los triglicéridos sin afectar al colesterol-HDL y los efectos fueron mayores en sujetos con colesterol basal más alto. Sin embargo, la participación de las isoflavonas como factor de protección cardiovascular no fue reconocido por la FDA, a pesar de los trabajos pioneros de Clarkson, que demostraron convincentemente que las isoflavonas contribuían a bajar el nivel sérico de colesterol ${ }^{38}$. En un estudio se comparó el efecto de caseína con aislados de proteína de soya que contienen entre 3 y $62 \mathrm{mg}$ de isoflavonas por $25 \mathrm{~g}$ de proteína. Se demostró que comparado con la caseína, $25 \mathrm{~g} /$ día de proteína de soya con $62 \mathrm{mg}$ de isoflavonas, como parte de una dieta baja en colesterol y grasas saturadas, reduce el colesterol total y colesterolLDL en individuos con hipercolesterolemia leve ${ }^{39}$. No se modificaron las concentraciones de triglicéridos ni colesterol-HDL.

En opinión de algunos investigadores y también en el nuestro, el potencial real de los alimentos que contienen isoflavonas estaría en la prevención de las ECV más que en su tratamiento. En este aspecto, puede ser beneficioso el consumo de proteína de soya aun en cantidades menores a las recomendadas de $25 \mathrm{~g}$ por día. $\mathrm{Al}$ respecto, la ingesta de proteína de soya en individuos japoneses adultos es en promedio de 6-8 gr por día y se encuentra que el nivel de colesterol en suero en adultos se correlaciona inversamente con la ingesta de proteína de soya $^{40}$.

Generalmente se acepta que la mujer en todas las edades, en comparación con el hombre, está relativamente protegida contra las ECV y muchos estudios sugieren que los estrógenos reducen los riesgos cardiovasculares. Se han propuesto varios mecanismos para explicar los efectos cardioprotectores de los estrógenos, incluyendo cambios asociados al metabolismo de los lípidos. Un metaanálisis sugiere que los estrógenos ejercen efectos favorables sobre las lipoproteínas, los factores de coagulación, sobre la distribución regional de las grasas, sobre el tiempo de oxida- 
ción de las LDL y efecto protector sobre las paredes arteriales, entre las cuales estaría la producción de moléculas vasoactivas tales como óxido nítrico y prostaglandinas ${ }^{41}$. Sin embargo, la sustitución estrogénica en la mujer posmenopáusica está asociada con un incremento en el riesgo de trombosis venosa ${ }^{42}$; más aún, el tratamiento con estrógenos en mujeres que tienen ECV en curso, aumenta la incidencia de infarto al miocardio y trombosis venosa ${ }^{43}$.

En el reciente estudio Framingham en mujeres posmenopáusicas, se relacionó la ingesta de fitoestrógenos (isoflavonas y lignanos) con un perfil metabólico favorable de menor riesgo cardiovascular $^{44}$. Los estudios realizados con la administración de isoflavonas en forma de suplementos, son controversiales para demostrar efectos sobre los niveles del colesterol circulante ${ }^{45,46}$. En la acción de las isoflavonas las propiedades no hormonales pueden ser de gran relevancia en la reducción de los riesgos para las enfermedades cardiovasculares. Varios estudios clínicos muestran que las isoflavonas reducen la susceptibilidad de los lípidos a la oxidación ${ }^{25,47}$ y, más aún, se ha observado que pueden tener efectos similares a la digitalina en la relajación de las arterias coronarias

\section{REFERENCIAS}

1. Adlercreutz H. Western diet and Western diseases: some hormonal and biochemical mechanism and associations. Scand J Clin Lab Invest 1990; 50: 201: 2-23.

2. Setchell KDR. Phytoestrogen: the biochemistry, physiology, and implication for human health of soy isoflavone. Am J Clin Nutr 1998; 68: 1333S1346S.

3. Tham DM, Gardner D, Haskell W. Potential health benefits of dietary phytoestrogen: A review of clinical epidemiological, and mechanism evidence. J Clin Endocrinol Metab 1998; 83: 222335.

4. WiLet WC. Balancing life-style and genomic research for disease prevention. Science 2002; 296: 695-8.

5. SETCHeLL KDR, CASSIDY A. Dietary isoflavones: biological effects and relevance to human health. J Nutr 1999; 129: 758S-67S. a través de un mecanismo que involucra un antagonismo de los canales de calcio ${ }^{48}$.

\section{CONCLUSIONES}

Hay evidencias insuficientes para recomendar cantidades específicas o tipos de isoflavonas para la prevención o tratamiento de determinadas enfermedades. Por esto, puede ser prematuro recomendar el reemplazo de la terapia de sustitución de estrógenos por isoflavonas de soya o de otras plantas. Las evidencias actuales no permiten obtener una clara conclusión dada las múltiples variables involucradas, como tamaño de la muestra, variabilidad en el diseño de los estudios, variación en los tipos y cantidades de las isoflavonas usadas. Aunque las evidencias para demostrar los efectos beneficiosos de los fitoestrógenos han incrementado notablemente (aproximadamente 600 manuscritos en el año 2002) aún se requiere de estudios más concluyentes. La recomendación más prudente es comer una dieta alta en cereales, frutas, vegetales y fibras, baja en carnes rojas y contenido de ácidos grasos saturados. Esto es consistente con las actuales recomendaciones y podría llevar a incrementar la ingesta diania de isoflavonas.

6. Murphy Pa, Song T, Buseman G, Barua K, Baecher GR. Isoflavones in retail and institutional soy foods. J Agric Food Chem 1999; 47: 2697-704.

7. Wang HJ, MurPHY PA. Isoflavone composition of American and Japanese soybean in Iowa: effect of variety, crop year and location. J Agric Food Chem 1994; 42: 1674-7.

8. Sfakianos J, Coward L, KirK M, Barnes S. Intestinal uptake and biliary excretion of the isoflavone genistein in rats. J Nutr 1997; 127: 1260-8.

9. AderRCReUtZ H. Epidemiology of phytoestrogen. Ballieres Clin Endocrinol Metabol 1998; 12: 60523.

10. Pino AM, Valadanees L, Palma M, Mancila A, Yánez M, Albala C. Dietary isoflavones affect sex hormone - binding globulin levels in postmenopausal women. J Clin Endocrinol Metab 2000; 85: 2797-800.

11. Chin-Dusting JP, Fisher LJ, Lewis TV, PiekArska A, Nestel PJ, Husband A. The vascular activity of some isoflavone metabolites: implications for a 
cardio protective role. Br J Pharmacology 2001; 133: 595-605.

12. Adiercreutz H, MarkKanen H, Watanabe S. Plasma concentration of phyto-oestrogens in Japanese men. Lancet 1993; 342: 1209-10.

13. Segura V. Efecto de un hábito de alimentación vegetariano sobre la biodisponibilidad de isoflavonas administradas a través de un suplemento. Tesis de Magíster en Ciencias de la Nutrición, INTA, Universidad de Chile. 2002.

14. Setchell KDR, Brown NM, Desai P, ZimmerNechemias L, Wolfe EB, Brashear WT et al. Bioavailability of pure isoflavones in healthy humans and analysis of commercial soy isoflavone supplements. J Nutr 2001; 131: 1362S-75S.

15. Olson JA, Moon RC, Ander MW. Enhancement of biological activity by conjugation reaction. J Nutr 1992; 122: 615-24.

16. Zhang Y, Song TT, Cunning JE, Murphy PA, HENDRICH S. Daidzein and genistein glucoronides in vitro are weakly estrogenic and activate human natural killer cells at nutritionally relevant concentration. J Nutr 1999; 129: 399-405.

17. LoNaRd D, SMTth CL Molecular perspectives on selective estrogen receptor modulators (SERMs); progress in understanding their tissue-specific agonist and antagonist actions. Steroids 2002; 67: 15-24.

18. Kuiper GG, Carlsson B, Grandien K, Enmark E, Haggblad J, NiLSSON S et al. Comparison of the ligand binding specificity and transcript tissue distribution of estrogen alpha and beta. Endocrinology 1997; 138: 863-70.

19. Barkhem T, CarlsSon B, NiLSSON Y, EnMark E, Gustafsson J, NiLsson S. Differential response of estrogen receptor alpha and estrogen receptor beta to partial estrogen agonist/antagonists. Mol Pharmacol 1998; 54: 105-12.

20. Webi P, López GN, Uth RM, Kushner PJ. Tamoxifen activation of the estrogen receptor /AP-1 pathway: potential origin for the cell-specific estrogen like effects of antiestrogens. Molec Endocrinol 1995; 9: 443-56.

21. Pike AC, Brzozowski AM, Hubbard ET. Structure of the ligand-binding domain of estrogen receptor beta in the presence of a partial agonist and a full antagonist. EMBO 1995; 18: 4608-18.

22. BRzezinski A. Phytoestrogen: the "natural" selective estrogen receptor modulators? European J Obstetrics Gynecol Reprod Biol 1999; 85: 47-51.
23. Kirk CJ, HarRis RM, Wood DM, Waring RH, Hughes TJ. Do dietary phytoestrogen influence susceptibility to hormone-dependent cancer by disrupting the metabolism of endogenous oestrogens? Biochem Soc Trans 2001; 29: 209-16.

24. Mousavi Y, Adlercreutz H. Genistein is an effective stimulator of SHBG production in hepatocarcinoma human liver cells and suppresses proliferation of these cells in culture. Steroid 1993; 58: 301-4.

25. Tikkanen MJ, Wahala K, Ohala S, Vihma V, AdierCREUTZ H. Effect of soybean phytoestrogen intake on low-density lipoprotein oxidation resistance. Proc Natl Acad Sci USA 1998; 95: 3106-10.

26. Messina M, Gugger ET, Alekel DL Soy protein, soybean isoflavones, and bone health: a review of the animal and human data. In: Handbook of Nutraceuticals and Functional Food: Ed. Wildman R. CRC Press, Boca Raton, FL 2001; 77-98.

27. Fournier DB, ERdMAN JWJ, Gordon GB. Soy, its components, and cancer prevention: a review of the in vitro, animal and human data. Cancer Epidemiol Biomarkers Prev 1998; 7: 1055-65.

28. Akiyama T, Ogawara $H$. Use and specificity of genistein as inhibitors of protein-tyrosine kinase. Methods Enzymol 1991; 201: 362-70.

29. Shu XO, Jin F, Dai Q, Wen W, PotTer JD, Kushi LH et AL. Soy food intake during adolescence and subsequent risk of breast cancer among Chinese women. Cancer Epidemiol Biomark Prev 2001; 10: 483-8.

30. Messina MJ, LopRinzi CL Soy for breast cancer survivors: A critical review of the literature. J Nutr 2001; 131: 3095S-3108S.

31. Cummings SR, Eckert S, Krueger KA, Grandy D, Powles TJ, Cauley JA et al. The effect of raloxifene on risk of breast cancer in postmenopausal women. Results from the MORE randomized trial. JAMA 1999; 281: 2189-97.

32. Etinger M, Genant HK, Cann CE. Long term estrogen replacement therapy prevents bone loses and fractures. Ann Intern Med 1985; 102: 319-24.

33. Kardinaal AF, Morton MS, Bruggemann-Rotnans IE, VAn BerestSeij EC. Phytoestrogen excretion and rate of bone loss in postmenopausal women. Eur J Clin Nutr 1998; 52: 850-5.

34. Arjmandi BH, Alekel L, Hows BW. Dietary soybean protein prevents bone loss in an ovariectomized rat model of osteoporosis. J Nutr 1996; 126: $160-5$. 
35. REGINSTER JY. Ipriflavone: pharmacological properties and rate of bone loss in postmenopausal osteoporosis. Bone Mineral 1993; 23: 223-32.

36. Ganbacciani M, Ciaponi M, Cappagli, Ganazzani AR. Effect of combined low dose of the isoflavone derivative ipriflavone and estrogen replacement on bone mineral density and metabolism in postmenopausal women. Maturitas 1997; 28: 7581.

37. Anderson JW, Johnstone BM, Cook-Newell ME. Meta-analysis of the effects of soy protein intake on serum lipids. N Engl J Med 1995; 333: 276-82.

38. Anthony MS, Clarkson TB, Hughes C. Plant and mammalian estrogen effects of soy protein intake on plasma lipid of female monkey. Circulation 1995; 90: 227-35.

39. Crouse Jr III, Morgan T, Terry JG, Eus J, Vitolns M, BuRKe GL. A randomized trial comparing the effect of casein with that of soy protein containing varying amounts of isoflavones on plasma concentrations of lipids and lipoproteins. Arch Intern Med 1999; 159: 2070-6.

40. NaKata C, TaKatsuka $N$, Kurisu $\mathrm{Y}$, Shimizu $\mathrm{H}$. Decreased serum total cholesterol concentration is associated with high intake of soy product in Japanese men and women. J Nutr 1998; 128: 20913.

41. Barred-Connor E, Bush TL. Estrogen and coronary heart disease in women. JAMA 1991; 265: 1861-7.

42. Varas-Lorenzo C, García-Rodríguez LA, Cattaruzzi C, Troncon MG, Agostinis L, Pérez-Gutthann S. Hormone replacement therapy and the risk of hospitalization for venous thromboembolism: a population-based study in Southern Europe. Am J Epidemiol 1998; 147: 387-90.

43. Pérez-Gutthann S, García Rodríguez LA, CastelSague J, Duque-O therapy and risk of venous thromboembolism population based case-control study. BMJ 1997; 15; 314: 796-800.

44. De Kieijn MJJ, Van der Schouw YT, WiLson DEG, JACQUES PF. Dietary intake of phytoestrogen is associated with a favorable metabolic cardiovascular risk profile in postmenopausal US women: The Framingham study. J Nutr 2002; 132: 276-82.

45. Nestel PJ, Yamashita T, Sasahara T, Pomeroy S, Dart A, Komesaroff P ET al. Soy isoflavones improve systemic arterial compliance but not plasma lipids in menopausal and perimenopausal women. Arterioscl Thromb Vascul Biol 1997; 17: 3392-8.

46. Hodgson JM, Puddey IB, Beilin LJ, Mori TA, Croft KD. Supplementation with isoflavonoid phytoestrogens does not alter serum lipid concentrations: a randomized controlled trial in humans. J Nutr 1998; 128: 728-32.

47. Wiseman H, O’Reiluy J, Adlercreutz H, Mauet A, Howey E, RowLAND I. Isoflavones phytoestrogen consumed in soy decrease f2- isoprostane concentration and increase resistance of low-density lipoprotein to oxidation in human. Am J Clin Nutr 2000; 72: 395-400.

48. Figree GA, Griffthis H, Lu Y-Q, Webb CM, MAcLeod K, Cowns P. Plant derived estrogen relax coronary arteries in vitro by a calcium antagonist mechanism. J Amer Coll Cardiol 2000; 35: 197785. 